\title{
DIGITALCOMMONS
}

$@$ WAYNESTATE-

Wayne State University

9-1-2007

\section{Necessary Conditions for Super Minimizers in Constrained Multiobjective Optimization}

Truong Q. Bao

Wayne State University

Boris S. Mordukhovich

Wayne State University, boris@math.wayne.edu

\section{Recommended Citation}

Bao, Truong Q. and Mordukhovich, Boris S., "Necessary Conditions for Super Minimizers in Constrained Multiobjective Optimization" (2007). Mathematics Research Reports. Paper 53.

http://digitalcommons.wayne.edu/math_reports/53 
NECESSARY CONDITIONS FOR SUPER MINIMIZERS IN CONSTRAINED MULTIOBJECTIVE OPTIMIZATION

TRUONG Q. BAO and BORIS S. MORDUKHOVICH

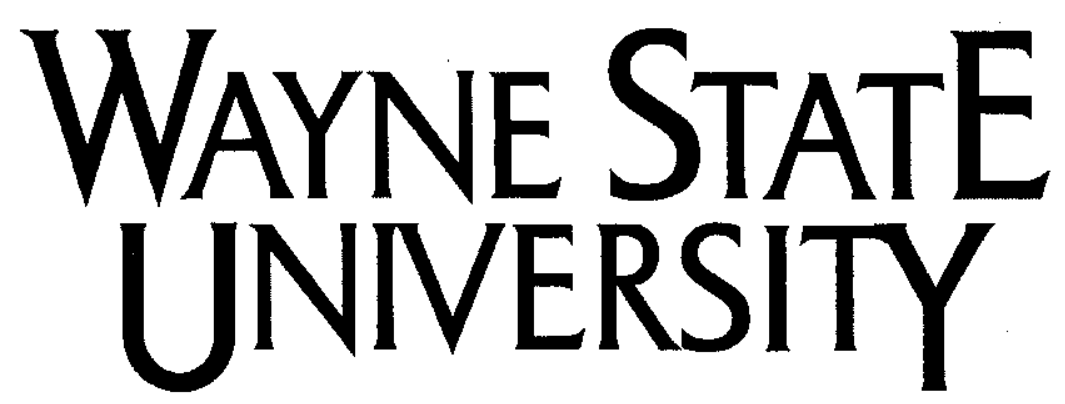

Detroit, MI 48202

Department of Mathematics

Research Report

2007 Series

\#9 


\title{
Necessary Conditions for Super Minimizers in Constrained Multiobjective Optimization
}

\author{
Truong Q. Bao and Boris S. Mordukhovich \\ Department of Mathematics, \\ Wayne State University, Detroit, MI48202 \\ Email: tqbao@math.wayne.edu, boris@math.wayne.edu
}

\begin{abstract}
This paper concerns the study of the so-called super minimizers related to the concept of super efficiency in constrained problems of multiobjective optimization, where cost mappings are generally set-valued. We derive necessary conditions for super minimizers on the base of advanced tools of variational analysis and generalized differentiation that are new in both finite-dimensional and infinite-dimensional settings for problems with single-valued and set-valued objectives.
\end{abstract}

Keywords Variational analysis - Nonsmooth and multiobjective optimization - Super efficiency and super minimizers · Generalized differentiation · Necessary optimality conditions

Mathematics Subject Classification (2000) 90C29 $\cdot 49 \mathrm{~J} 52 \cdot 49 \mathrm{~J} 53$

\section{Introduction}

This paper is devoted to constrained problems of multiobjective optimization given by:

$$
\text { minimize } F(x) \text { subject to } x \in \Omega \subset X \text {, }
$$

where the cost mapping $F: X \rightrightarrows Z$ between Banach spaces is generally set-valued, and where "minimization" is understood with respect to some partial ordering on $Z$. In this setting, (1.1) is a problem of constrained set-valued optimization, while the term "vector optimization" is conventionally used when $F=f: X \rightarrow Z$ is a single-valued mapping. Therefore, we utilize the name of "multiobjective optimization" to unify both classes of set-valued and vector optimization problems under consideration.

Let $Z$ be a partially ordered Banach space, where the partial order is generated by a nonempty cone $\Theta \neq \emptyset$, which we always assume to be closed and convex while not generally pointed. Denoting the ordering relation on $Z$ by " $\leq$ ", we have:

$$
z_{1} \leq z_{2} \quad \text { if and only if } \quad z_{2}-z_{1} \in \Theta
$$

Given a nonempty subset $\Xi \subset Z$ and a point $\bar{z} \in \Xi$, we recall the classical notion of vector optimality: $\bar{z} \in \Xi$ is a minimal point of $\Xi$, known also as a Pareto optimal point or as an efficient point of $\Xi$, if

$$
\Xi \cap(\bar{z}-\Theta)=\{\bar{z}\} \text { or, equivalently, }(\Xi-\bar{z}) \cap(-\Theta)=\{0\} .
$$

Considering now the multiobjective optimization problem (1.1) defined via a set-valued mapping $F: X \rightrightarrows Z$ with the graph

$$
\operatorname{gph} F:=\{(x, z) \in X \times Z \mid z \in F(x)\},
$$


we say as usual that $(\bar{x}, \bar{z}) \in \operatorname{gph} F$ is a local minimizer to (1.1) if $\bar{x} \in \Omega$ and there is a neighborhood $U$ of $\bar{x}$ such that $\bar{z}$ is a minimal point of the image set

$$
\Xi=F(\Omega \cap U):=\bigcup_{x \in \Omega \cap U} F(x)
$$

which means by definition (1.3) that

$$
F(\Omega \cap U) \cap(\bar{x}-\Theta)=\{\bar{z}\}
$$

If $U=Z$ in (1.4), then the point $(\bar{x}, \bar{z})$ is called a minimizer to problem (1.1).

There are various modifications of the above efficiency notion intensively studied in the literature; see, e.g., the books $[4,10,11,14,16]$ with the references and discussions therein. A lot of attention has been paid to the weak counterpart of definition (1.4) related to the usage of certain scalarization techniques involving eventually applications of convex separation theorems and their variants; see $[10,11,14]$ for more details. On the other hand, it has been well recognized that the weak efficiency/weak Pareto optimality and associated scalarization techniques lead to serious limitations from the viewpoints of adequate descriptions of vector optimization problems and practical applications. To eliminate some of these disadvantages, the notion of proper efficiency was introduced by Geoffrion [9] following the pioneering work by Kuhn and Tucker [13]; see also the subsequent papers by Borwein [6] and Benson [5] for significant improvements of proper efficiency.

In this paper we pay the main attention to the notion of super efficiency introduced by Borwein and Zhuang [8] and then studied in many publications; see, e.g., $[8,12,20]$ and the references therein. This notion refines and/or unifies various modifications of proper efficiency and reflects crucial features of solutions to vector optimization problems important from the viewpoints of both the theory and applications. We refer the reader to [8] for various characterizations of super efficiency and its relationships with other solution notions in multiobjective optimizations and economics.

The primary goal of this paper is to derive verifiable necessary conditions for super efficiency using modern variational principles and variational techniques together with advanced constructions of generalized differentiation. Our approach is mainly based on the extremal principle of variational analysis, which can be viewed as a variational counterpart of the local separation principle in nonconvex settings; see the books $[15,16]$ for all the details on the extremal principle and its numerous applications to various problems of scalar and vector optimization, economics, control theory, etc. We also refer the reader to more recent papers $[1,2,3]$, where the extremal principle and the corresponding results of the well-developed generalized differential calculus generated by the extremal principle are applied to deriving the existence theorems and necessary optimality/suboptimality conditions for efficient and weakly efficient solutions to multiobjective problems with general and structural constraints. In particular, it is proved in [3] that the condition

$$
0 \in \partial F(\tilde{x}, \bar{z})+N(\check{x} ; \Omega)
$$

is necessary for local efficient points/minimizers to problem (1.1), and it is also necessary for weak local minimizers to (1.1) provided that int $\Theta \neq \emptyset$. To formulate (1.5), we use the appropriate notions of generalized normals to sets and subgradients of set-valued mappings defined and discussed below in Section 2. In this paper we use these and related generalized 
differential constructions to obtain new necessary optimality conditions for super minimizers to the general constrained multiobjective problem (1.1) and its specifications.

The rest of the paper is organized as follows. In Section 2 we present and briefly discuss some major tools of variational analysis and generalized differentiation widely used in formulating and proving the main results. Section 3 is devoted to deriving qualified necessary optimality conditions for super minimizers to (1.1) under general assumptions on the initial data expressed in terms of coderivatives of the cost mapping $F$. We also present in this section new necessary conditions for super minimal points of sets. In Section 4 we establish relationships between coderivatives and subdifferentials of set-valued mappings, which are certainly of independent interest, and then derive subdifferential conditions for super minimizers to multiobjective problems. The subdifferential conditions are generally independent of the coderivative ones from Section 3 and require additional assumptions on the initial data, while on the other hand they improve the latter in many important settings. The major results obtained in both Section 3 and Section 4 are illustrated by examples and are compared with those known in the literature.

Throughout the paper we use standard notation of variational analysis; cf. $[15,19]$. Recall that $\mathbb{N}:=\{1,2, \ldots\}$ and that $\mathbb{B}$ and $\mathbb{B}^{*}$ stand, respectively, for the closed unit balls in the Banach space in question and its topological dual. Given a set-valued mapping $F: X \rightrightarrows X^{*}$ between a Banach $X$ and its dual $X^{*}$, the symbol

$$
\begin{aligned}
\operatorname{Limsup}_{x \rightarrow \bar{x}} F(x):=\left\{x^{*} \in X^{*} \mid\right. & \exists \text { sequences } x_{k} \rightarrow \bar{x} \text { and } x_{k}^{*} \stackrel{w^{*}}{\rightarrow} x^{*} \\
& \text { with } \left.x_{k}^{*} \in F\left(x_{k}\right) \text { for all } k \in \mathbb{N}\right\}
\end{aligned}
$$

signifies the sequential Painlevé-Kuratowski upper/outer limit of $F$ at $\bar{x}$ in the norm topology of $X$ and weak* topology $w^{*}$ of $X^{*}$.

\section{Basic Tools of Variational Analysis}

In this section we briefly overview some basic generalized differential constructions and related notions of variational analysis widely used in formulations and proofs of our main results of the paper. We follow the recent books by Mordukhovich $[15,16]$, where the reader can find more details, discussions and references. We also refer the reader to the now classical book by Rockafellar and Wets [19] in finite dimensions and to the recent book by Borwein and Zhu [7] in Fréchet smooth spaces for related and additional material on variational analysis, generalized differentiation, and their applications.

Let $\Omega \subset X$ be a subset of a Banach space, and let $\bar{x} \in \Omega$. The (basic, limiting, Mordukhovich) normal cone to $\Omega$ at $\bar{x}$ is defined by

$$
N(\bar{x} ; \Omega):=\operatorname{Limsup}_{\substack{x \rightarrow \bar{x} \\ \varepsilon \downarrow 0}} \widehat{N}_{\varepsilon}(\bar{x} ; \Omega)
$$

via the sequential Painlevé-Kuratowski outer limit (1.6) of the sets of $\varepsilon$-normals

$$
\widehat{N}_{\varepsilon}(x ; \Omega):=\left\{x^{*} \in X^{*} \mid \limsup _{u \stackrel{\Omega}{\longrightarrow}} \frac{\left\langle x^{*}, u-x\right\rangle}{\|u-x\|} \leq \varepsilon\right\}, \quad x \in \Omega,
$$

where $u \stackrel{\Omega}{\rightarrow} x$ means that $u \rightarrow x$ with $u \in \Omega$, and where $\widehat{N}_{\varepsilon}(x ; \Omega):=\emptyset$ whenever $x \notin \Omega$ and $\varepsilon \geq 0$. When $\varepsilon=0$, the construction (2.2) is known as the Fréchet/regular normal cone 
to $\Omega$ at $x$. Furthermore, we can equivalently put $\varepsilon=0$ in (2.1) if the space $X$ is Asplund while the set $\Omega$ is locally closed around $x$.

Recall that a Banach space $X$ is Asplund if any convex continuous function is densely Fréchet differentiable on $X$. This class is sufficiently broad including, in particular, all reflexive Banach spaces and Banach spaces with separable duals. There are many equivalent descriptions of Asplund spaces that can be found, e.g., in $[7,15]$ and the references therein.

In contrast to the sets of $\varepsilon$-normals (2.2), the normal cone (2.1) is nonconvex in common finite-dimensional and infinite-dimensional situations enjoying nevertheless full calculus in Asplund spaces and partly in the general Banach space setting; see [15] for a comprehensive study based on the extremal principle and related tools of variational analysis.

Given a set-valued mapping $F: X \rightrightarrows Z$ between Banach spaces, consider two coderivatives of $F$ at the point $(\bar{x}, \bar{z}) \in \operatorname{gph} F$ : the normal coderivative

$$
D_{N}^{*} F(\bar{x}, \bar{z})\left(z^{*}\right):=\left\{x^{*} \in X^{*} \mid\left(x^{*},-z^{*}\right) \in N((\bar{x}, \bar{z}) ; \operatorname{gph} F)\right\}
$$

and the mixed coderivative of $F$ at $(\bar{x}, \bar{z})$ defined by

$$
\begin{aligned}
& D_{M}^{*} F(\bar{x}, \bar{z})\left(z^{*}\right):=\left\{x^{*} \in X^{*} \mid \exists \varepsilon_{k} \downarrow 0,\left(x_{k}, z_{k}\right) \stackrel{\operatorname{gph} F}{\rightarrow}(\bar{x}, \bar{z}), x_{k}^{*} \stackrel{w^{*}}{\rightarrow} x^{*}, z_{k}^{*} \rightarrow z^{*}\right. \\
& \text { with } \left.\left(x_{k}^{*},-z_{k}^{*}\right) \in \widehat{N}_{\varepsilon}\left(\left(x_{k}, z_{k}\right) ; \operatorname{gph} F\right), \quad k \in \mathbb{N}\right\} \text {, }
\end{aligned}
$$

where one can equivalently put $\varepsilon_{k}=0$ if both spaces $X$ and $Z$ are Asplund and if the graph of $F$ is locally closed around $(\bar{x}, \bar{z})$. We always omit $\bar{z}$ in these coderivative notation if $F=f: X \rightarrow Z$ is single-valued. It easily follows from (2.1), (2.3), and (2.4) above that

$$
D_{M}^{*} F(\bar{x}, \bar{z})\left(z^{*}\right) \subset D_{N}^{*} F(\bar{x}, \tilde{z})\left(z^{*}\right) \text { for all } z^{*} \in Z^{*},
$$

where equality holds and we use the common symbol $D^{*} F(\bar{x}, \bar{y})$ for both coderivatives when, in particular, $\operatorname{dim} Z<\infty$. Moreover, we have

$$
D_{N}^{*} f(\bar{x})\left(z^{*}\right)=D_{M}^{*} f(\bar{x})\left(z^{*}\right)=\left\{\nabla f(\bar{x})^{*} z^{*}\right\} \text { for all } z^{*} \in Z^{*}
$$

if $f: X \rightarrow Z$ is strictly differentiable at $\bar{x}$ with the derivative $\nabla f(\bar{x})$, i.e.,

$$
\lim _{x, u \rightarrow \bar{x}} \frac{f(x)-f(u)-\langle\nabla f(\bar{x}), x-u\rangle}{\|x-u\|}=0,
$$

which is automatic when $f \in C^{1}$ around this point.

Let us now consider the case of a mapping $F: X \rightrightarrows Z$ between Banach spaces with the range space $Z$ partially ordered via (1.2) by a closed and convex cone $\Theta \subset Z$. Define the epigraph of $F$ with respect to the ordering cone $\Theta$ by

$$
\text { epi } F:=\{(x, z) \in X \times Z \mid z \in F(x)+\Theta\}
$$

and the associated epigraphical multifunction $\mathcal{E}_{F}: X \rightrightarrows Z$ by

$$
\mathcal{E}_{F}(x):=\{z \in Z \mid z \in F(x)+\Theta\} .
$$

Given $(\bar{x}, \bar{z}) \in$ epi $F$, we define the basic/normal subdifferential of $F$ at the point $(\ddot{x}, \bar{z})$ in the direction $z^{*} \in Z^{*}$ by

$$
\partial F(\bar{x}, \bar{z})\left(z^{*}\right):=D_{N}^{*} \mathcal{E}_{F}(\bar{x}, \bar{z})\left(z^{*}\right)
$$


This construction relates to the normal subdifferential of $F$ at $(\ddot{x}, \bar{z})$ introduced in [2] by

$$
\partial F(\bar{x}, \bar{z}):=\left\{x^{*} \in X^{*} \mid x^{*} \in D_{N}^{*} \mathcal{E}_{F}(\bar{x}, \bar{z})\left(z^{*}\right), \quad-z^{*} \in N(0 ; \Theta),\left\|z^{*}\right\|=1\right\} .
$$

If $\varphi: X \rightarrow \overline{\mathbb{R}}:=(-\infty, \infty]$ is an extended-real-valued function finite at $\bar{x}$ with $\bar{z}=\varphi(\bar{x})$, the subdifferential construction (2.7) with $\Theta=\mathbb{R}_{+}$reduces to the basic/limiting subdifferential by Mordukhovich; see [15] and the references therein. In Section 4 we establish new relationships between the subdifferentials of set-valued mappings $F$ with values in partially ordered spaces and coderivatives of such mappings versus coderivatives of the associated epigraphical multifunctions.

In what follows we also need the singular subdifferential of $F$ at $(\bar{x}, \bar{z})$ defined by

$$
\partial^{\infty} F(\bar{x}, \bar{z}):=D_{M}^{*} \mathcal{E}_{F}(\bar{x}, \bar{z})(0),
$$

which brings nontrivial information only for mappings that do not exhibit a certain Lipschitzian behavior. Namely, we have

$$
\partial^{\infty} F(\bar{x}, \bar{z})=\{0\}
$$

if $F$ is epi-Lipschitz-like (ELL) around $(\tilde{x}, \bar{z}) \in$ epi $F$ in the sense that there are neighborhoods $U$ of $\bar{x}$ and $V$ of $\bar{z}$ and a number $\ell \geq 0$ such that

$$
\mathcal{E}_{F}(x) \cap V \subset \mathcal{E}_{F}(u)+\ell\|x-u\| \text { whenever } x, u \in U .
$$

One of the most important ingredients of variational analysis in infinite dimensions is the necessity to impose some "normal compactness" properties, which are automatic in finite dimensions while compensate the lack of compactness in infinite-dimensional spaces. Let us recall some of such properties, which are needed in this paper; see $[15,16]$ for a comprehensive theory (including well-developed calculus/preservation rules) and applications mainly based on the extremal principle.

Given a set-valued mapping $F: X \rightrightarrows Y$ between Banach spaces, we say that $F$ is sequentially normally compact (SNC) at $(\bar{x}, \bar{z}) \in \operatorname{gph} F$ if for any sequences of elements $\left(\varepsilon_{k}, x_{k}, z_{k}, x_{k}^{*}, z_{k}^{*}\right) \in \mathbb{R} \times X \times Z \times X^{*} \times Z^{*}$ satisfying

$$
\varepsilon_{k} \downarrow 0, \quad\left(x_{k}, z_{k}\right) \stackrel{\Omega}{\rightarrow}(\bar{x}, \bar{z}), \quad \text { and }\left(x_{k}^{*}, z_{k}^{*}\right) \in \widehat{N}_{\varepsilon_{k}}\left(\left(x_{k}, z_{k}\right) ; \operatorname{gph} F\right), \quad k \in \mathbb{N},
$$

one has the implication

$$
\left(x_{k}^{*}, z_{k}^{*}\right) \stackrel{w^{*}}{\rightarrow}(0,0) \Longrightarrow\left\|\left(x_{k}^{*}, z_{k}^{*}\right)\right\| \rightarrow 0 \text { as } k \rightarrow \infty,
$$

while $\varepsilon_{k}$ can be equivalently omitted in (2.11) if $X$ and $Z$ are Asplund spaces and if the graph of $F$ is locally closed around $(\bar{x}, \bar{z})$.

A set $\Omega \subset Z$ is $S N C$ at $\bar{z} \in \Omega$ if the constant mapping $F \equiv \Omega$ has this property. In [15], the reader can find a number of efficient conditions ensuring the fulfillment of the SNC property of sets and mappings (in particular, the so-called "compactly epi-Lipschitzian property" in the sense of Borwein and Strójwas.). In the case of mappings, the following partial modification of the SNC property happens to be more appropriate for many applications including those in this paper: $F: X \rightrightarrows Z$ is partially $S N C$ (PSNC) at $(\bar{x}, \bar{z}) \in \operatorname{gph} F$ if for any sequences satisfying (2.11) we have the implication

$$
\left[x_{k}^{*} \stackrel{w^{*}}{\rightarrow} 0, \quad\left\|z_{k}^{*}\right\| \rightarrow 0\right] \Longrightarrow\left\|x_{k}^{*}\right\| \rightarrow 0 \text { as } k \rightarrow \infty
$$


In particular, the latter property is automatic if $F$ is Lipschitz-like (or "pseudo-Lipschitz" in the sense of Aubin) around $(\bar{x}, \bar{z})$, which is defined as in (2.10) with the replacement of $\mathcal{E}_{F}$ by $F$ and seems to be the most natural extension of Lipschitzian behavior to set-valued mappings; see $[15,19]$ for more details and discussions.

Considering next a mapping $F: X \rightarrow Z$ whose range space is partially ordered by the ordering cone $\Theta \subset Z$, we use in what follow an appropriate modification of the PSNC property. The mapping $F$ is said to be partially sequentially normally epi-compact (partially SNEC) at $(\bar{x}, \bar{z}) \in$ epi $F$ if the epigraphical multifunction $\mathcal{E}_{F}$ is PSNC at this point. The latter holds for any mapping $F$ that is ELL around $(\bar{x}, \bar{z})$.

\section{Coderivative Conditions for Super Minimizers in Multiob- jective Problems}

This section is devoted to establishing general qualified necessary optimality conditions for super minimizers to the constrained multiobjective optimization problem (1.1) in terms of the normal and mixed coderivatives of the set-valued or single-valued cost mapping $F$ and the limiting normals to the constraint set $\Omega$ defined in Section 2 .

First we recall the notion of super minimal points to arbitrary subsets of partially ordered spaces introduced by Borwein and Zhuang [8]. Given a subset $\Xi$ of a Banach space $Z$ ordered via (1.2) by a closed and convex cone $\Theta \subset Z$, we say that $\bar{z} \in \Xi$ is a super minimal point of $\Xi$ if there is a number $M>0$ such that

$$
\operatorname{cl}[\text { cone }(\Xi-\bar{z})] \cap(\mathbb{B}-\Theta) \subset M \mathbb{B}
$$

where "cone" stands for the conic hull spanned on the set $\Xi-\bar{z}$, with the subsequent closure "cl", and where $\mathbb{B}$ signifies the closed unit ball of $Z$. It is easy to see that (3.1) can be equivalently expressed as:

$$
\|z-\bar{z}\| \leq M\|v\| \text { for any } z \in \Xi \text { and } v \in Z \text { satisfying } z-\bar{z} \leq v .
$$

It is immediately implied by (1.3) and (3.1) that every super minimal point of $\Xi$ is surely a minimal/efficient/Pareto point of this set. The notion of super minimal points for sets naturally induces the following definition of super minimizers to constrained multiobjective problems in form (1.1), which is the main object of our study in this paper.

Definition 3.1 (super minimizers to multiobjective problems) Let $(\bar{x}, \bar{z}) \in \operatorname{gph} F$ with $\bar{x} \in \Omega$. Then $(\bar{x}, \bar{z})$ is a LOCAL SUPER MINIMIZER to problem (1.1) if there is a neighborhood $U$ of $\bar{x}$ such that $\bar{z}$ is a super minimal point of the image set

$$
\Xi:=F(\Omega \cap U)=\bigcup_{x \in \Omega \cap U} F(x)
$$

which can be equivalently written as follows: there is $M>0$ such that

$$
\|z-\bar{z}\| \leq M\|v\| \text { whenever } x \in \Omega \cap U, z \in F(x), v \in Z \text { with } z-\bar{z} \leq v .
$$

As always, $(\bar{x}, \bar{z})$ is a SUPER MINIMIZER to (1.1) if we can choose $U=X$ in (3.2). 
Our first theorem provides qualified necessary optimality conditions for super minimizers to problem (1.1) in terms of the normal coderivative (2.3) to $F$ and the limiting normal cone (2.1) to $\Omega$, with the qualification condition expressed via the mixed coderivative (2.4) of the cost mapping. On the other hand, in the proof of this theorem we use some well-known subdifferential constructions for extended-real-valued functions and certain important results for them recalled in what follows.

Given $\varphi: X \rightarrow \overline{\mathbb{R}}$ finite at $\bar{x}$ and given $\varepsilon \geq 0$, define the $\varepsilon$-subdifferential of $\varphi$ at $\bar{x}$ by

$$
\widehat{\partial}_{\varepsilon} \varphi(\bar{x}):=\left\{x^{*} \in X^{*} \mid \liminf _{x \rightarrow \bar{x}} \frac{\varphi(x)-\varphi(\bar{x})-\left\langle x^{*}, x-\bar{x}\right\rangle}{\|x-\bar{x}\|} \geq-\varepsilon\right\} .
$$

For $\varepsilon=0$, construction (3.3) is known as the Fréchet/regular subdifferential of $\varphi$ at $\bar{x}$ and is denoted by $\widehat{\partial} \varphi(\bar{x})$. It is well known that the latter subdifferential $\widehat{\partial} \varphi(\bar{x})$ reduces to the classical subdifferential of convex analysis for convex functions $\varphi$, while it may be empty for simple nonconvex functions as, e.g., for $\varphi(x)=-|x|$ at $0 \in \mathbb{R}$.

Another disadvantage of (3.3) is a poor pointwise calculus; e.g., the standard sum rule $\widehat{\partial}\left(\varphi_{1}+\varphi_{2}\right)(\bar{x}) \subset \widehat{\partial} \varphi_{1}(\bar{x})+\widehat{\partial} \varphi_{2}(\bar{x})$ does not hold in elementary situations. Recently the following rather surprising sum rule

$$
\widehat{\partial}\left(\varphi_{1}+\varphi_{2}\right)(\bar{x}) \subset \bigcap_{x^{*} \widehat{\partial}^{+} \varphi_{1}(\bar{x})}\left[x^{*}+\widehat{\partial} \varphi_{2}(\bar{x})\right]
$$

has been discovered in [17] for arbitrary extended-real-valued functions on Banach spaces provided that the upper counterpart of the Fréchet subdifferential

$$
\widehat{\partial}^{+} \varphi(\bar{x}):=-\widehat{\partial}(-\varphi)(\bar{x})
$$

of $\varphi_{1}$ at $\bar{x}$ is nonempty in (3.4); see $[15,17]$ for verifiable conditions ensuring this property.

Another subdifferential construction used in what follows is the (basic, limiting, Mordukhovich) subdifferential of $\varphi: X \rightarrow \overline{\mathbb{R}}$ finite at $\bar{x}$ defined by

$$
\partial \varphi(\bar{x}):=\underset{\substack{x \rightarrow \varphi \\ \varepsilon \downarrow 0}}{\operatorname{Limsup}} \widehat{\partial}_{\varepsilon} \varphi(x),
$$

where the symbol $x \stackrel{\varphi}{\rightarrow} \bar{x}$ stands for $x \rightarrow \bar{x}$ with $\varphi(x) \rightarrow \varphi(\bar{x})$, and where we can equivalently put $\varepsilon=0$ if the space $X$ is Asplund and if the function $\varphi$ is lower semicontinuous (l.s.c.) around $\bar{x}$. We have the basic subdifferential sum rule

$$
\partial\left(\varphi_{1}+\varphi_{2}\right)(\bar{x}) \subset \partial \varphi_{1}(\bar{x})+\partial \varphi_{2}(\bar{x})
$$

provided that the $X$ is Asplund and that $\varphi_{1}$ is Lipschitz continuous around $\bar{x}$ while $\varphi_{2}$ is l.s.c. around this point; see [15, Theorem 2.33].

Now we are ready to formulate and prove the main coderivative necessary conditions for super minimizers to the constrained multiobjective problem (1.1).

Theorem 3.2 (general necessary coderivative conditions for super minimizers). Let $(\bar{x}, \bar{z}) \in \operatorname{gph} F$ be a local super minimizer to problem (1.1), where the spaces $X$ and $Z$ are Asplund, and where $Z$ is partially ordered via (1.2) by a closed and convex cone $\Theta \subset Z$. Assume that the graph of $F$ and the set $\Omega$ are locally closed around $(\bar{x}, \bar{z})$ and $\bar{x}$, respectively, and that the cone $\Theta$ is $S N C$ at the origin. Furthermore, impose the requirements: 
(a) either $F$ is PSNC at $(\bar{x}, \bar{z})$, or $\Omega$ is SNC at $\bar{x}$;

(b) the qualification condition on $\{F, \Omega\}$ is satisfied:

$$
D_{M}^{*} F(\bar{x}, \bar{z})(0) \cap(-N(\bar{x} ; \Omega))=\{0\},
$$

which both hold automatically when $F$ is Lipschitz-like around $(\bar{x}, \bar{z})$.

Then taking $M>0$ from Definition 3.1 of local super minimizers and taking any $v^{*}$ from the closed unit ball of $Z^{*}$, we find $-z^{*} \in N(0 ; \Theta)$ with $\left\|z^{*}\right\| \leq M$ such that

$$
0 \in D_{N}^{*} F(\bar{x}, \bar{z})\left(z^{*}-v^{*}\right)+N(\bar{x} ; \Omega) .
$$

Proof. Given a local super minimizer $(\bar{x}, \bar{z})$ to problem (1.1), fix a neighborhood $U$ of $\bar{x}$ and a number $M>0$ from Definition 3.1 such that relationship (3.2) is satisfied. Consider the distance function $d_{\Theta}: Z \rightarrow \mathbb{R}$ to the closed and convex set $\bar{z}-\Theta \subset Z$ omitting $\bar{z}$ in the notation of $d_{\Theta}$ for simplicity. Fix $z \in Z$ and take any $\gamma>0$. Then there is $\theta \in \Theta$ such that

$$
\|v\| \leq d_{\Theta}(z)+\gamma \text { for } v:=z-\bar{z}+\theta
$$

Since $z-\bar{z} \leq v$ by (1.2) and since $\gamma>0$ is chosen arbitrarily in (3.9), we conclude from (3.9) and the underlying super minimizer relationship (3.2) that

$$
\|z-\bar{z}\| \leq M d_{\Theta}(z) \text { for all } z \in F(x) \text { with } x \in \Omega \cap U .
$$

To proceed further, we build three subsets of $X \times Z$ by

$$
\Lambda_{1}:=\operatorname{gph} F, \quad \Lambda_{2}:=\Omega \times Z, \quad \Lambda:=\Lambda_{1} \cap \Lambda_{2}
$$

and define an extended-real-valued function $\varphi: X \times Z \rightarrow \overline{\mathbb{R}}$ by

$$
\varphi(x, z):=-\|z-\bar{z}\|+M d_{\Theta}(z)+\delta((x, z) ; \Lambda),
$$

where $\delta(\cdot ; \Lambda)$ is the indicator function of $\Lambda$ equal to 0 on $\Lambda$ and to $\infty$ on its complement.

It easily follows from (3.10)-(3.12) that $(\bar{x}, \bar{z})$ is a local minimizer to $\varphi$ over $X \times Z$. Then the generalized Fermat rule from [15, Proposition 1.114] gives

$$
0 \in \widehat{\partial} \varphi(\bar{x}, \bar{z})=\widehat{\partial}\left[-\|\cdot-\bar{z}\|+M d_{\Theta}(\cdot)+\delta(\cdot ; \Lambda)\right](\bar{x}, \bar{z}) .
$$

Employing now the Fréchet subdifferential sum rule (3.4) to the functions

$$
\varphi_{1}(x, z):=-\|z-\bar{z}\| \text { and } \varphi_{2}(x, z):=M d_{\Theta}(z)+\delta((x, z) ; \Lambda)
$$

in (3.13) and taking into account that

$$
\widehat{\partial}^{+}(-\|\cdot-\bar{z}\|)(\bar{x}, \bar{z})=-\widehat{\partial}(\|\cdot-\bar{z}\|)(\bar{x}, \bar{z})=\{0\} \times \mathbb{B}^{*}
$$

via the closed unit ball $\mathbb{B}^{*}$ in $Z^{*}$, we obtain in this way that

$$
\widehat{\partial}\left[-\|\cdot-\bar{z}\|+\left(M d_{\Theta}(\cdot)+\delta(\cdot ; \Lambda)\right)\right](\bar{x}, \bar{z}) \subset \bigcap_{z^{*} \in \mathbb{B}^{*}}\left[\left(0, z^{*}\right)+\widehat{\partial}\left(M d_{\Theta}(\cdot)+\delta(\cdot ; \Lambda)\right)(\bar{x}, \bar{z})\right] .
$$

The latter inclusion implies by (3.13) that

$$
\{0\} \times \mathbb{B}^{*} \subset \widehat{\partial}\left(M d_{\Theta}(\cdot)+\delta(\cdot ; \Lambda)\right)(\bar{x}, \bar{z}) \subset \partial\left(M d_{\Theta}(\cdot)+\delta(\cdot ; \Lambda)\right)(\bar{x}, \bar{z}) .
$$


Since the product space $X \times Z$ is Asplund, we apply the basic subdifferential sum rule (3.6) to the semi-Lipschitzian sum in (3.14) and get the inclusion

$$
\{0\} \times \mathbb{B}^{*} \subset \partial\left(M d_{\Theta}(\cdot)\right)(\bar{x}, \bar{z})+\partial \delta((\bar{x}, \bar{z}) ; \Lambda)=\{0\} \times M \partial d_{\Theta}(\bar{z})+\partial \delta((\bar{x}, \bar{z}) ; \Lambda),
$$

where the last representation is due to $d_{\Theta}(\cdot)=d_{\Theta}(z)$. Taking now into account that $\delta((\bar{x}, \bar{z}) ; \Lambda))=N\left((\bar{x}, \bar{z}) ; \Lambda_{1} \cap \Lambda_{2}\right)$ and that

$$
\partial d_{\Theta}(\bar{z})=\mathbb{B}^{*} \cap N(\bar{z} ; \bar{z}-\Theta)=\mathbb{B}^{*} \cap(-N(0 ; \Theta))
$$

by $[15$, Theorem 1.96$]$, we get from (3.15) that

$$
\{0\} \times \mathbb{B}^{*} \subset\{0\} \times\left[M \mathbb{B}^{*} \cap(-N(0 ; \Theta))\right]+N\left((\bar{x}, \bar{z}) ; \Lambda_{1} \cap \Lambda_{2}\right) .
$$

To proceed further in (3.16), we employ the intersection rule for basic normals from $[15$, Theorem 3.4] to the intersection $\Lambda_{1} \cap \Lambda_{2}$ of the sets $\Lambda_{1}$ and $\Lambda_{2}$ defined in (3.11). It is easy to check that assumption (a) of the theorem and structures (3.11) of the sets $\Lambda_{i}$ ensure that either $\Lambda_{1}$ is PSNC at $(\bar{x}, \bar{z})$ with respect to $X$ and the other set $\Omega_{2}$ is strongly PSNC at this point with respect to $Z$, or $\Lambda_{2}$ is SNC at $(\bar{x}, \bar{z})$. It also follows from the structures of the set $\Lambda_{1}$ and $\Lambda_{2}$ that the qualification condition (3.7) in terms of the mixed coderivative of the cost mapping $F$ implies the limiting qualification condition imposed in [15, Theorem 3.4]. Thus we get the inclusion

$$
N\left((\bar{x}, \bar{z}) ; \Lambda_{1} \cap \Lambda_{2}\right) \subset N\left((\bar{x}, \bar{z}) ; \Lambda_{1}\right)+N\left((\bar{x}, \bar{z}) ; \Lambda_{2}\right) .
$$

Substituting finally (3.17) into (3.16) with taking into account the structures of $\Lambda_{i}$ and definition (2.3) of the normal coderivative, we arrive at the necessary condition (3.8) with $-z^{*} \in N(0 ; \Theta)$ and $\left\|z^{*}\right\| \leq M$ and thus complete the proof of the theorem.

Remark 3.3 (qualified form of optimality conditions). Note that the necessary conditions for super minimizers obtained in Theorem 3.2 are of qualified form provided that $\Theta \neq\{0\}$. The latter means that we do not have

$$
z^{*}-v^{*}=0 \text { for all } v^{*} \in \mathbb{B}^{*} \text { with }-z^{*} \in N(0 ; \Theta)
$$

in (3.8). Indeed, the opposite immediately yields that

$$
\mathbb{B}^{*} \subset N(0 ; \Theta)=Z^{*}
$$

and therefore $\Theta=\{0\}$, a contradiction.

Before deriving other necessary conditions for super minimizers to the general constrained multiobjective problem (1.1) and its specifications, let us present two simple examples illustrating the application of the results obtained in Theorem 3.2, their comparison with the corresponding necessary conditions for Pareto minimizers and weak Pareto minimizers, and also discuss the relationships of the results obtained for super minimizers with those known in the literature.

Example 3.4 (illustration of necessary conditions for super minimizers). Consider problem (1.1) with $\Theta=\mathbb{R}_{+}^{2}$ (the nonnegative orthant of $\mathbb{R}^{2}$ ), $\Omega=\mathbb{R}$, and the constant set-valued mapping $F: \mathbb{R} \rightrightarrows \mathbb{R}^{2}$ given by

$$
F(x) \equiv \Xi \text { with } \Xi:=\left\{\left(z_{1}, z_{2}\right) \in \mathbb{R}^{2} \mid z_{1}+2 z_{2} \geq 0 \text { or } 2 z_{1}+z_{2} \geq 0\right\}
$$


It is easy to check that $(\bar{x}, \bar{z})=(0,0) \in \mathbb{R} \times \mathbb{R}^{2}$ is a super minimizer to the problem under consideration; actually it is a super minimal point of the set $\Xi$ in (3.18). All the assumptions of Theorem 3.2 are trivially satisfied. By definition of the basic normal cone (2.1) we have

$$
\begin{gathered}
N\left(0 ; \mathbb{R}_{+}^{2}\right)=\mathbb{R}_{-}^{2} \text { and } N((0,0) ; \text { epi } F)=\{0\} \times \Lambda, \quad \text { where } \\
\Lambda:=\left\{\left(z_{1}, z_{2}\right) \in \mathbb{R}_{-}^{2} \mid 2 z_{1}-z_{2}=0 \text { or }-z_{1}+2 z_{2}=0\right\} .
\end{gathered}
$$

Since $\mathbb{R}_{+}^{2}+\Lambda=\mathbb{R}^{2}$, for any $v$ from the closed unit ball of $\mathbb{R}^{2}$ we find $u \in \mathbb{R}^{2}$ with $-u \in N\left(0 ; \mathbb{R}_{+}^{2}\right)=\mathbb{R}_{-}^{2}$ such that $v-u \in \Lambda$. Thus

$$
0 \in D^{*} F(0,0)(v-u)=D^{*} F(0,0)(v-u)+N(0 ; \mathbb{R})
$$

for the vectors $v$ and $u$ under consideration, and the optimality condition (3.8) is satisfied.

The next example shows that the necessary condition (1.5) for minimizers and weak minimizers to problem (1.1) recently obtained in $[2,3]$ does not provide a necessary condition for super minimizers.

Example 3.5 (comparison with necessary conditions for minimizers and weak minimizers). Consider problem (1.1) with $\Theta=\mathbb{R}_{+}^{2}, \Omega=\mathbb{R}$, and the set-valued cost mapping $F: \mathbb{R} \Rightarrow \mathbb{R}^{2}$ given by

$$
F(x) \equiv \mathbb{R}^{2} \backslash \mathbb{R}_{+}^{2} \text { with epi } F=\operatorname{gph} F .
$$

It is easy to see that $(\bar{x}, \bar{z})=(0,0) \in \mathbb{R} \times \mathbb{R}^{2}$ is a weak minimizer to the problem (1.1) under consideration (actually it is a weak efficient point of the set $\mathbb{R}^{2} \backslash$ (int $\mathbb{R}_{+}^{2}$ )) while it is not a super minimizer to this problem. Let us check the fulfillment of the necessary conditions (1.5) and (3.8). We directly compute the corresponding coderivative and subdifferential used in (1.5) and (3.8). Indeed, we have

$$
\begin{gathered}
N((0,0) ; \operatorname{epi} F)=N((0,0) ; \operatorname{gph} F)=\{0\} \times \text { bd } \mathbb{R}_{-}^{2}, \\
D^{*} F(0,0)\left(u_{1}, u_{2}\right)=\partial F(0,0)\left(u_{1}, u_{2}\right)= \begin{cases}0 & \text { if } u_{1}=0 \text { and } u_{2} \geq 0 \\
0 & \text { if } u_{2}=0 \text { and } u_{1} \geq 0 \\
\emptyset & \text { otherwise }\end{cases}
\end{gathered}
$$

and so the necessary condition (1.5) is satisfied for $(0,0)$. Taking now arbitrary vectors

$$
v:=\left(v_{1}, v_{2}\right)=-\left(\frac{\sqrt{2}}{2}, \frac{\sqrt{2}}{2}\right) \text { and }-u:=\left(-u_{1},-u_{2}\right) \in N\left(0 ; \mathbb{R}_{+}^{2}\right)=\mathbb{R}_{-}^{2},
$$

we immediately arrive at the following relationships for these vectors:

$$
u_{1}-v_{1}>0, \quad u_{2}-v_{2}>0, \text { and } D^{*} F(0,0)(u-v)=\emptyset,
$$

which show that the condition (3.8) is not satisfied. This confirms by Theorem 3.2 that $(0,0)$ is not a super minimizer to the problem (1.1) under consideration. 
Remark 3.6 (comparison with known conditions for super minimizers). Quite recently, Huang [12] has obtained necessary conditions for super minimizers to problem (1.1) that are expressed in terms of the Clarke generalized differential constructions and extend previous results in this direction; see [12] for more discussions and references. Taking into account the relationship between the Clarke normal cone and our basic normal cone from [15, Theorem 3.57], the main necessary conditions derived in [12] can be written in the form of Theorem 3.2 with the replacement of (3.8) by

$$
0 \in \bar{D}^{*} F(\bar{x}, \bar{z})\left(z^{*}-v^{*}\right)+\operatorname{cl}^{*} \operatorname{co} N(\bar{x} ; \Omega)
$$

where "cl*" co" stands for the convex closure of a dual space subset in the corresponding weak* topology, and where we use the notation

$$
\bar{D}^{*} F(\bar{x}, \bar{z})\left(u^{*}\right):=\left\{x^{*} \in X^{*} \mid\left(x^{*},-u^{*}\right) \in \mathrm{cl}^{*} \operatorname{co} N((\bar{x}, \bar{z}) ; \operatorname{gph} F)\right\} .
$$

The necessary conditions of [12] are justified under certain tangential qualification assumptions and interiority-type requirements, which are significantly more restrictive than the qualification assumption (3.7) and the PSNC/SNC properties imposed in Theorem 3.2. We are not going to discuss these relationships in more detail here while focusing only on the comparison between necessary optimality conditions (3.8) and (3.19).

It turns out that condition (3.19) is not just trivially implied by (3.8) but the convexification operation in (3.20) may dramatically enlarge the first set on the right-hand side of (3.8)-as much as often getting there the whole space-in many situations important for both the theory and application. More precisely, for any locally Lipschitzian single-valued mapping $F: X \rightarrow Z$ the convexified normal cone in (3.20) is always a linear subspace of $X^{*} \times Z^{*}$ whose dimension is not less than $m$ if $Z=\mathbb{R}^{m}$; see $[15$, Theorem 3.62 and Corollary 3.67]. Moreover, these facts hold not only for graphs of single-valued locally Lipschitzian mappings but also for set-valued mappings whose graphs are Lipschitzian manifolds, or hemi-Lipschitzian sets; see [15, Theorem 3.72]. The latter objects include maximal monotone operators and subdifferential mappings for convex and major classes of nonconvex (e.g., prox-regular) functions typically encountered in variational analysis and optimization; see more details and discussions in [15, Subsection 1.2.2 after Definition 1.45]. When both spaces $X$ and $Z$ are finite-dimensional, the afore-mentioned results go back to the seminal paper by Rockafellar [18]. These discussions reveal therefore crucial limitations of the optimality condition (3.19) in comparison with the new one in (3.8).

A visible disadvantage of the optimality condition (3.8) in Theorem 3.2 is that it involves all elements $v^{*}$ from the unit ball of $Z^{*}$ and does not constructively specify the choice of the corresponding normal $-z^{*} \in N(0 ; \Theta)$. The next theorem establishes a new version of necessary conditions for super minimizers to (1.1) that is more verifiable and convenient for applications. Its proof requires, however, the additional interiority assumption

$$
\text { int } N(0 ; \Theta) \neq \emptyset
$$

imposed on the ordering cone $\Theta$. Note that assumption (3.21) does not require that int $\Theta \neq \emptyset$ and automatically holds provided that the cone $\Theta$ is closed and convex with a bounded base, i.e., when there is a bounded convex set $\Theta_{B} \subset Z$ such that

$$
\Theta=\mathbb{R}_{+} \Theta_{B} \text { and } 0 \notin \operatorname{cl} \Theta_{B} \text {. }
$$


Theorem 3.7 (verifiable conditions for super minimizers under the interiority assumption). Let $(\bar{x}, \bar{z}) \in \operatorname{gph} F$ be a local super minimizer to problem (1.1) under all the assumptions of Theorem 3.2. Suppose in addition that $\Theta \neq\{0\}$ and that the interiority requirement (3.21) is satisfied. Then there is $-z^{*} \in \operatorname{int} N(0 ; \Theta)$ with $\left\|z^{*}\right\|=1$ such that

$$
0 \in D_{N}^{*} F(\bar{x}, \bar{z})\left(z^{*}\right)+N(\bar{x} ; \Omega) .
$$

Proof. It immediately follows from the set inclusion (3.16) justified in Theorem 3.2 that

$$
\{0\} \times \mathbb{B}^{*} \subset\{0\} \times(-N(0 ; \Theta))+N((\bar{x}, \bar{z}) ; \Lambda)
$$

with the closed unit ball $\mathbb{B}^{*} \subset Z^{*}$ and the intersection set $\Lambda$ defined in (3.11). Denoting

$$
C^{*}:=\left\{z^{*} \in Z^{*} \mid\left(0, z^{*}\right) \in N((\bar{x}, \bar{z}) ; \Lambda)\right\},
$$

we have from (3.23) that $\mathbb{B}^{*} \subset C^{*}-N(0 ; \Theta)$ and hence the space $Z^{*}$ admits the representation $Z^{*}=C^{*}-N(0 ; \Theta)$, since the latter sets are cones. Using this decomposition of $Z^{*}$ and the interiority assumption of the theorem, pick an element $u^{*} \in \operatorname{int} N(0 ; \Theta)$ and represent it as $u^{*}=c^{*}-p^{*}$ with $c^{*} \in C^{*}$ and $p^{*} \in N(0 ; \Theta)$. Thus

$$
c^{*}=u^{*}+p^{*} \in \operatorname{int} N(0 ; \Theta)+N(0 ; \Theta) \subset \operatorname{int} N(0 ; \Theta) \text {. }
$$

It follows from $(3.25)$ that $c^{*} \neq 0$; otherwise $0 \in \operatorname{int} N(0 ; \Theta)$ and hence $N(0 ; \Theta)=Z^{*}$, which contradicts the assumption $\Theta \neq\{0\}$ of the theorem. Letting now $z^{*}:=-c^{*} /\left\|c^{*}\right\|$, we get from the structures of $C^{*}$ in (3.24) and $\Lambda$ in (3.11) that

$$
\left(0,-z^{*}\right) \in N\left((\bar{x}, \bar{z}) ; \Lambda_{1} \cap \Lambda_{2}\right) \text { with }-z^{*} \in \operatorname{int} N(0 ; \Theta) \text { and }\left\|z^{*}\right\|=1 .
$$

Employing finally the intersection rule (3.17) justified in the proof of Theorem 3.2, we arrive from (3.26) to all the conclusions of this theorem.

Note that the new necessary condition (3.22) is more convenient to deal with in comparison with the previous condition (3.8) from Theorem 3.2. To illustrate this, consider Example 3.5. It immediately follows from the explicit formula for computing the coderivative presented above that there is no $-z^{*} \in \operatorname{int} N(0 ; \Theta)=\operatorname{int} \mathbb{R}_{-}^{2}$ satisfying (3.22). Thus $(\bar{x}, \bar{z})=(0,0)$ is not a super minimizer to the multiobjective problem under consideration.

Finally in this section, we present new necessary conditions for super minimal points of sets that follow from Theorem 3.2 and Theorem 3.7.

Corollary 3.8 (necessary conditions for super minimal points of sets). Let $Z$ be an Asplund space partially ordered by a closed and convex cone $\Theta \subset Z$ that is $S N C$ at the origin. If $\bar{z} \in \Xi$ is a super minimal point of the set $\Xi \subset Z$ locally closed around $\bar{z}$, then for any $v^{*}$ from the closed unit ball $\mathbb{B}^{*}$ of $Z^{*}$ there is $z^{*} \in N(0 ; \Theta)$ such that

$$
z^{*}-v^{*} \in N(\bar{z} ; \Xi) \text { and }\left\|z^{*}\right\| \leq M
$$

where $M>0$ is taken from definition (3.1) of the super minimal point. If furthermore int $N(0 ; \Theta) \neq \emptyset$, than there is $z^{*} \in Z^{*}$ with $\left\|z^{*}\right\|=1$ such that

$$
z^{*} \in N(\bar{z} ; \Xi) \text { and } z^{*} \in \operatorname{int} N(0 ; \Theta) \text {. }
$$


Proof. The first assertion of the corollary involving condition (3.27) follows from Theorem 3.2 applied to the constant mapping $F(x) \equiv \Xi$ and $\Omega=X$. It is easy to see that (3.27) follows from the coderivative condition (3.8) due to the product formula

$$
\left(0,-z^{*}\right) \in N((\bar{x}, \bar{z}) ; \operatorname{gph} F)=\{0\} \times N(\bar{z} ; \Xi) .
$$

In the same way condition (3.28) follows from condition (3.22) of Theorem 3.7.

Comparing condition (3.28) for super minimal points with condition (1.5) for minimal and weak minimal ones and taking into account the subdifferential construction (2.3) in (1.5), observe that the main difference between the necessary conditions for super minimal and minimal/weak minimal points is that we get $-z^{*}$ belonging to the interior of $N(0 ; \Theta)$ in the first case in comparison with just $-z^{*} \in N(0 ; \Theta)$ in the second one. Furthermore, the necessary condition (3.28) for super minimal points to $\Xi$ yields

$$
N(\bar{z} ; \Xi)-N(0 ; \Theta)=Z^{*}
$$

due to int $N(0 ; \Theta) \neq \emptyset$, which obviously implies the condition

$$
0 \in \operatorname{int}\left(-\mathrm{cl}^{*} \operatorname{co} N(0 ; \Theta)+\mathrm{cl}^{*} \operatorname{co} N(\bar{z} ; \Xi)\right)
$$

established in [20] under significantly more restrictive assumptions.

Finally in this section, let us illustrate the efficiency of the more involved necessary condition (3.27) for super minimal points in the situation when condition (3.28) is not applicable. Consider the ordering cone $\Theta \subset \mathbb{R}^{2}$ given by

$$
\Theta:=\left\{z \in \mathbb{R}^{2} \mid z_{1} \geq 0\right\},
$$

which is obviously not pointed with the empty interior of the normal cone

$$
N(0 ; \Theta)=\left\{z \in \mathbb{R}^{2} \mid z_{1} \leq 0, z_{2}=0\right\} .
$$

Taking the set $\Xi:=\Theta$ and the point $0 \in \Xi$, we cannot employ condition (3.28) to check the super minimality of this point. On the other hand, choosing $\left(v_{1}, v_{2}\right)=(0,1) \in \mathbb{B}$, we have $z_{2}=0$ for any $\left(z_{1}, z_{2}\right) \in N(0 ; \Theta)$, and hence $\left(z_{1}-v_{1}, z_{2}-v_{2}\right)=\left(z_{1}, 1\right) \notin N(0 ; \Xi)$. Condition (3.27) allows us to conclude that 0 is not a super minimal point of the set $\Xi$ with respect to the ordering cone $\Theta$ under consideration.

\section{Coderivative-Subdifferential Relationships and Subdiffer- ential Conditions for Super Minimizers}

The primary goal of this section is to derive necessary conditions of the subdifferential type for super minimizers to the original multiobjective problem (1.1). We derive subdifferential conditions under some additional assumptions in comparison with the coderivative ones in Section 3 and establish relationships between these two types of necessary conditions for super minimizers to (1.1). To proceed, we first discuss relationships between coderivatives and subdifferentials of general set-valued mappings between Banach spaces for which the range spaces are partially ordered by a closed and convex cone. The results obtained in this direction are certainly of independent interest.

The following property of set-valued and single-valued mappings with partially ordered range spaces generalizes the classical lower semicontinuity of extended-real-valued functions being important for deriving coderivative-subdifferential relationships. 
Definition 4.1. (order semicontinuity of set-valued mappings). Let $F: X \rightrightarrows Z$ be $a$ set-valued mapping between two Banach spaces, where the range space $Z$ is partially ordered via (1.2) by a closed and convex cone $\Theta \subset Z$. We say that $F$ is ORDER SEMICONTINUOUs at $(\bar{x}, \bar{z}) \in \operatorname{gph} F$ if for any sequence $\left\{\left(x_{k}, z_{k}\right)\right\} \subset$ epi $F$ there is a sequence $\left\{\left(x_{k}, v_{k}\right)\right\} \subset \operatorname{gph} F$ with $v_{k} \leq z_{k}$ such that $\left\{v_{k}\right\}$ contains a subsequence converging to $\bar{z}$.

It is easy to see that, besides lower semicontinuous extended-real-valued functions, this class contains every single-valued mapping $f: X \rightarrow Z$ continuous at $\bar{x}$. Let us present a general condition ensuring the fulfillment of order semicontinuity for a large class of setvalued mappings at minimal/efficient points of the corresponding image sets. Recall that by (1.3) the minimal set to $\Xi$ with respect to the ordering cone $\Theta$ is described by

$$
\text { Min } \Xi:=\{\bar{z} \in \Xi \mid \bar{z}-z \notin \Theta \text { whenever } z \in \Xi, z \neq \bar{z}\} \text {. }
$$

Proposition 4.2 (sufficient conditions for order semicontinuity). Let $F: X . \rightrightarrows Z$ be closed-graph and locally compact around $\bar{x} \in \operatorname{dom} F$ in the setting of Definition 4.1. Then it is order semicontinuous at $(\bar{x}, \bar{z})$ for every $\bar{z} \in \operatorname{Min} F(\bar{x})$.

Proof. Fix $\bar{z} \in \operatorname{Min} F(\bar{x})$ and take any sequence $\left\{\left(x_{k}, z_{k}\right)\right\} \subset$ epi $F$ converging to $(\bar{x}, \bar{z})$. By definition of the epigraph epi $F$, select a sequence $\left\{v_{k}\right\}$ satisfying

$$
v_{k} \in F\left(x_{k}\right) \text { and } v_{k} \leq z_{k} \text { for all } k \in \mathbb{N} \text {. }
$$

By the assumed local compactness of $F$ around $\bar{x}$, we suppose without loss of generality that the sequence $\left\{v_{k}\right\}$ converges to some point $\bar{v}$, which belongs to $F(\bar{x})$ due to the closed-graph property of $F$ around $\bar{x}$. To justify the order semicontinuity of $F$ at $(\bar{x}, \bar{z})$, it remains to show that $\bar{v}=\bar{z}$. To proceed, we suppose that $\bar{v} \not z \bar{z}$, i.e., that

$$
\bar{v}-\bar{z} \in Z \backslash(-\Theta) \text {. }
$$

Since the complement $Z \backslash(-\Theta)$ is an open subset of $Z$, there is a number $\eta>0$ such that

$$
(\bar{v}+\eta \mathbb{B})-(\bar{z}+\eta \mathbb{B}) \subset Z \backslash(-\Theta) .
$$

Taking into account that $z_{k} \rightarrow \bar{z}$ and $v_{k} \rightarrow \bar{v}$ as $k \rightarrow \infty$, we get from the last inclusion that

$$
v_{k}-z_{k} \in Z \backslash\{-\Theta\} \text { or, equivalently, } v_{k} \not z_{k}
$$

for all $k \in \mathbb{N}$ sufficiently large. The latter clearly contradicts (4.1) and thus shows that $\bar{v} \leq \bar{z}$. By the choice of $\bar{z} \in \operatorname{Min} F(\bar{x})$ we get therefore that $\bar{v}=\bar{z}$, which completes that proof of the proposition.

Observe that the choice of $\bar{z} \in \operatorname{Min} F(\bar{x})$ in Proposition 4.2 is essential for the validity of the order semicontinuity property of $F$ at $(\tilde{x}, \bar{z})$. To illustrate this, consider a mapping $F: \mathbb{R} \rightrightarrows \mathbb{R}$ defined by

$$
F(x):= \begin{cases}0 & \text { if } x \neq 0 \\ {[0,1]} & \text { if } x=0\end{cases}
$$

Taking $1 \in F(0) \backslash(\operatorname{Min} F(0))$, we check that $F$ is not order semicontinuous at $(0,1) \in \operatorname{gph} F$. Indeed, the sequence $\left\{\left(k^{-1}, 1\right)\right\} \subset$ epi $F$ converges to $(0,1)$ while the uniquely corresponding to it sequence from the graph $\left\{\left(k^{-1}, 0\right)\right\} \subset \operatorname{gph} F$ converges to $(0,0)$.

The above order semicontinuity property is crucial to establish the following relationships between the subdifferential (2.6) and the normal coderivative (2.3) of mappings in the corresponding directions. 
Proposition 4.3 (relationships between subdifferentials and normal coderivatives of mappings). Let $F: X \rightrightarrows Z$ be a mapping between Banach spaces, where $Z$ is partially ordered by a closed and convex cone $\Theta \subset Z$. Assume that $F$ is order semicontinuous at $(\bar{x}, \bar{z}) \in \operatorname{gph} F$. Then we have the inclusion

$$
\partial F(\bar{x}, \bar{z})\left(z^{*}\right) \subset D_{N}^{*} F(\bar{x}, \bar{z})\left(z^{*}\right) \text { for all } z^{*} \in Z^{*} .
$$

Proof. Using the subdifferential definition (2.6) and taking any $z^{*} \in Z^{*}$, we fix some subgradient $x^{*} \in \partial F(\bar{x}, \bar{z})\left(z^{*}\right):=D_{N}^{*} \mathcal{E}_{F}(\bar{x}, \bar{z})\left(z^{*}\right)$ and find by (2.3) and (2.1) sequences $\varepsilon_{k} \downarrow 0,\left(x_{k}, z_{k}\right) \rightarrow(\bar{x}, \bar{z})$, and $\left(x_{k}^{*}, z_{k}^{*}\right) \stackrel{w^{*}}{\rightarrow}\left(x^{*}, z^{*}\right)$ as $k \rightarrow \infty$ satisfying

$$
\left(x_{k}, z_{k}\right) \in \text { epi } F \text { and }\left(x_{k}^{*},-z_{k}^{*}\right) \in \widehat{N}_{\varepsilon_{k}}\left(\left(x_{k}, z_{k}\right) ; \text { epi } F\right), \quad k \in \mathbb{N} .
$$

By the order semicontinuity property of $F$ at $(\bar{x}, \bar{z})$, there is a sequence

$$
v_{k} \in F\left(x_{k}\right) \text { with } v_{k} \leq z_{k} \text { and } v_{k} \rightarrow \bar{z} \text { as } k \rightarrow \infty \text {, }
$$

where we take all $k \in \mathbb{N}$ without loss of generality. It follows from the second inclusion in (4.3) and definition (2.2) that for any $\gamma>0$ there is $\eta>0$ such that

$$
\left\langle\left(x_{k}^{*},-z_{k}^{*}\right),(x, z)-\left(x_{k}, z_{k}\right)\right\rangle \leq\left(\gamma+\varepsilon_{k}\right)\left\|(x, z)-\left(x_{k}, z_{k}\right)\right\|
$$

whenever $(x, z) \in \operatorname{epi} F, x \in x_{k}+\eta \mathbb{B}, z \in z_{k}+\eta \mathbb{B}$, and $k \in \mathbb{N}$. Using the construction of epi $F$ generated by the ordering cone $\Theta$, we get the relationship

$$
z_{k}=v_{k}+\theta_{k} \text { with some } \theta_{k} \in \Theta, \quad k \in \mathbb{N} \text {. }
$$

Taking further an arbitrary vector $(u, v) \in$ epi $F$ with $u \in x_{k}+\eta \mathbb{B}$ and $v \in v_{k}+\eta \mathbb{B}$, we find $\left(\widetilde{v}_{k}, \widetilde{\theta}_{k}\right) \in Z \times Z$ by the above epigraphical construction such that

$$
v=\widetilde{v}_{k}+\tilde{\theta}_{k} \text { with } \tilde{v}_{k} \in F(u) \text { and } \tilde{\theta}_{k} \in \Theta, \quad k \in \mathbb{N} .
$$

Now we define the vector $v_{k}:=v+\left(z_{k}-v_{k}\right)$ and get from (4.6), (4.7), and the convexity of the ordering cone $\Theta$ that

$$
v_{k}=\widetilde{v}_{k}+\widetilde{\theta}_{k}+\theta_{k} \in F(u)+\Theta, \quad k \in \mathbb{N} .
$$

Since $\left\|v_{k}-z_{k}\right\|=\left\|v-v_{k}\right\| \leq \eta$, we have by the construction of $v_{k}$ that

$$
\left(u, v_{k}\right) \in \operatorname{epi} F \text { with } u \in x_{k}+\eta \mathbb{B} \text { and } v_{k} \in z_{k}+\eta \mathbb{B} \text {. }
$$

Substituting now $\left(u, v_{k}\right)$ into the $\varepsilon_{k}$-normal relationship (4.5) and taking into account that $v_{k}-z_{k}=v-v_{k}$, we get

$$
\left\langle\left(x_{k}^{*},-z_{k}^{*}\right),(u, v)-\left(x_{k}, v_{k}\right)\right\rangle \leq\left(\gamma+\varepsilon_{k}\right)\left\|(u, v)-\left(x_{k}, v_{k}\right)\right\|, \quad k \in \mathbb{N} .
$$

Since $\gamma>0$ and $(u, v) \in\left(x_{k}, v_{k}\right)+\eta(\mathbb{B} \times \mathbb{B})$ were chosen arbitrarily and since the sets of $\varepsilon$-normals enjoy the monotonicity property $\widehat{N}_{\varepsilon}(\bar{y} ; \Omega) \subset \widehat{N}_{\varepsilon}(\bar{y} ; \widetilde{\Omega})$ whenever $\bar{y} \in \widetilde{\Omega} \subset \Omega$ and $\varepsilon \geq 0$, we arrive from (4.8) at the inclusions

$$
\left(x_{k}^{*},-z_{k}^{*}\right) \in \widehat{N}_{\varepsilon_{k}}\left(\left(x_{k}, v_{k}\right) ; \text { epi } F\right) \subset \widehat{N}_{\varepsilon_{k}}\left(\left(x_{k}, v_{k}\right) ; \operatorname{gph} F\right), \quad k \in \mathbb{N} .
$$

The latter gives, by (4.4) as $k \rightarrow \infty$ and definitions (2.1) and (2.3), that $x^{*} \in D_{N}^{*} F(\bar{x}, \bar{z})\left(z^{*}\right)$, which ensures (4.2) and completes the proof of the proposition.

The next proposition establishes relationships between the mixed coderivatives (2.4) of the mapping $F$ and the associated epigraphical multifunction (2.5), which implies the opposite inclusion in (4.2) when $\operatorname{dim} Z<\infty$. 
Proposition 4.4 (relationships between mixed coderivatives of mappings and the associated epigraphical multifunctions). Let $F: X \rightrightarrows Z$ be a set-valued mapping between Banach spaces under the assumptions of Proposition 4.3 fulfilled at some point $(\bar{x}, \bar{z}) \in \operatorname{gph} F$. Then we have the inclusion

$$
D_{M}^{*} F(\bar{x}, \bar{z})\left(z^{*}\right) \subset D_{M}^{*} \mathcal{E}_{F}(\bar{x}, \bar{z})\left(z^{*}\right) \text { whenever } \inf _{\theta \in \Theta} \frac{\left\langle z^{*}, \theta\right\rangle}{\|\theta\|}=: \nu>0 \text {. }
$$

Proof. Pick any $x^{*} \in D_{M}^{*} F(\bar{x}, \bar{z})\left(z^{*}\right)$, where $z^{*} \in Z^{*}$ satisfies the conditions in (4.9). By the mixed coderivative definition (2.4) we find sequences $\varepsilon_{k} \downarrow 0,\left(x_{k}, z_{k}\right) \rightarrow(\bar{x}, \bar{z}), x_{k}^{*} \stackrel{w^{*}}{\rightarrow} x^{*}$, and $z_{k}^{*} \rightarrow z^{*}$ as $k \rightarrow \infty$ such that

$$
\left(x_{k}, z_{k}\right) \in \operatorname{gph} F \text { and }\left(x_{k}^{*},-z_{k}^{*}\right) \in \widehat{N}_{\varepsilon_{k}}\left(\left(x_{k}, z_{k}\right) ; \operatorname{gph} F\right) \text { for all } k \in \mathbb{N} .
$$

For simplicity and without loss of generality, we can assume that $z_{k}^{*}=z^{*}$ for all $k \in \mathbb{N}$, since otherwise $\left(x_{k}^{*},-z^{*}\right) \in \widehat{N}_{\tilde{\varepsilon}_{k}}\left(\left(x_{k}, z_{k}\right) ; \operatorname{gph} F\right)$ with $\widetilde{\varepsilon}_{k}:=\varepsilon_{k}+\left\|z_{k}^{*}-z^{*}\right\| \rightarrow 0$ as $k \rightarrow \infty$. To justify (4.9), let us first show that

$$
\left(x_{k}^{*},-z^{*}\right) \in \widehat{N}_{\varepsilon_{k}}\left(\left(x_{k}, z_{k}\right) ; \text { epi } F\right) \text { for large } k \in \mathbb{N} .
$$

Arguing by contradiction, suppose that (4.11) does not hold for the chosen element $z^{*}$ satisfying the condition in (4.9) with some $\nu>0$ and a fixed number $k \in \mathbb{N}$ large enough to have $\nu-\varepsilon_{k}>0$. Then we find a number $0<\gamma<\nu-\varepsilon_{k}$ and a sequence $\left\{\left(u_{i}, v_{i}\right)\right\} \subset$ epi $F$ with $\left(u_{i}, v_{i}\right) \rightarrow\left(x_{k}, z_{k}\right)$ as $i \rightarrow \infty$ such that

$$
\left\langle\left(x_{k}^{*},-z^{*}\right),\left(u_{i}, v_{i}\right)-\left(x_{k}, z_{k}\right)\right\rangle>\left(\gamma+\varepsilon_{k}\right)\left\|\left(u_{i}, v_{i}\right)-\left(x_{k}, z_{k}\right)\right\|, \quad i \in \mathbb{N} .
$$

Using the order semicontinuity of $F$ similarly to the proof of Proposition 4.3, we find a sequence $\widetilde{v}_{i} \rightarrow z_{k}$ as $i \rightarrow \infty$ such that

$$
v_{i}=\widetilde{v}_{i}+\theta_{i} \text { and } \widetilde{v}_{i} \in F\left(u_{i}\right) \text { with some } \theta_{i} \in \Theta \text { as } i \in \mathbb{N} \text {. }
$$

If follows from the requirement on $z^{*}$ in (4.9) that $\left\langle z^{*}, \theta_{i}\right\rangle \geq \nu\left\|\theta_{i}\right\|$ for all $i \in \mathbb{I}$. Taking this into account and substituting $\left(u_{i}, \widetilde{v}_{i}\right)$ from (4.13) into (4.12), we get by $\nu>\gamma+\varepsilon_{k}$ that

$$
\begin{aligned}
& \left\langle\left(x_{k}^{*},-z^{*}\right),\left(u, \widetilde{v}_{i}\right)-\left(x_{k}, z_{k}\right)\right\rangle>\left(\gamma+\varepsilon_{k}\right)\left\|\left(u_{i}, \widetilde{v}_{i}+\theta_{i}\right)-\left(x_{k}, z_{k}\right)\right\|+\left\langle z^{*}, \theta_{i}\right\rangle \\
& \geq\left(\gamma+\varepsilon_{k}\right)\left\|\left(u_{i}, \widetilde{v}_{i}\right)-\left(x_{k}, z_{k}\right)\right\|+\nu\left\|\theta_{i}\right\|-\left(\gamma+\varepsilon_{k}\right)\left\|\theta_{i}\right\| \\
& \geq\left(\gamma+\varepsilon_{k}\right)\left\|\left(u_{i}, \widetilde{v}_{i}\right)-\left(x_{k}, z_{k}\right)\right\| \text { for all } i \in \mathbb{N}
\end{aligned}
$$

which means that $\left(x_{k}^{*},-z^{*}\right) \notin \widehat{N}_{\varepsilon_{k}}\left(\left(x_{k}, z_{k}\right)\right.$;gph $\left.F\right)$. The latter contradicts $(4.10)$ and thus justifies inclusion (4.11) for all $k \in \mathbb{N}$ sufficiently large, where we can replace $z^{*}$ by $z_{k}^{*} \rightarrow z^{*}$. Passing finally to the limit in (4.11) as $k \rightarrow \infty$ and taking into account definition (2.4) of the mixed coderivative for the epigraphical multifunction (2.5), we get $x^{*} \in D_{M}^{*} \mathcal{E}_{F}(\bar{x}, \bar{z})\left(z^{*}\right)$ and complete the proof of the proposition.

Let us present two corollaries of the results established in Proposition 4.3 and Proposition 4.4 ensuring the equality relationships between subdifferentials and coderivatives.

Corollary 4.5 (subdifferential-coderivative equality for set-valued mappings). Let $\operatorname{dim} Z<\infty$ under the assumptions of Proposition 4.3. Then

$$
\partial F(\bar{x}, \bar{z})\left(z^{*}\right)=D^{*} F(\bar{x}, \bar{z})\left(z^{*}\right) \text { whenever } \inf _{\theta \in \Theta} \frac{\left\langle z^{*}, \theta\right\rangle}{\|\theta\|}>0 .
$$


Proof. This follows directly from inclusions (4.2) and (4.9), since the normal and mixed coderivatives agree for mappings with finite-dimensional range spaces.

Corollary 4.5 easily implies the unconditional equality between the basic/limiting subdifferential and the corresponding coderivative of l.s.c. extended-real-valued functions on arbitrary Banach spaces. Note that, despite the range space of extended-real-valued functions $\varphi: X \rightarrow \mathbb{R}$ is not standard, the coderivative definition (2.3) involving only graphical points $\operatorname{gph} \varphi \subset X \times \mathbb{R}$ makes sense in this case.

Corollary 4.6 (subdifferential-coderivative equality for lower semicontinuous functions). Let $\varphi: X \rightarrow \overline{\mathbb{R}}$ be finite at $\bar{x}$ and l.s.c. around this point on the Banach space $X$. Then we have the equality

$$
\partial \varphi(\bar{x})=D^{*} \varphi(\bar{x})(1)
$$

Proof. Immediately follows from Corollary 4.5 with $\Theta=R_{+}$and $z^{*}=1$.

The result obtained in Corollary 4.6 extends the one from [15, Theorem 1.80] established for continuous real-valued functions. The example presented right after the proof of Theorem 1.80 in [15] shows that we do not have the equality $\partial^{\infty} \varphi(\bar{x})=D^{*} \varphi(\bar{x})(0)$ for the singular subdifferential of continuous functions $\varphi: \mathbb{R} \rightarrow \mathbb{R}$. This illustrates that the requirement on $z^{*}$ imposed in (4.14) is essential.

Let us derive new necessary conditions for super minimizers to the constrained multiobjective problem (1.1) expressed via subdifferentials of the cost mapping. The coderivativesubdifferential relationships obtained above allow us to compare these subdifferential conditions for super minimizers with the coderivative ones derived in Section 3.

In accordance with the conventional terminology in multiobjective optimization (see, e.g., $[11,14])$, we say that the ordering cone $\Theta \subset Z$ has the normality property if its combination $(\mathbb{B}+\Theta) \cap(\mathbb{B}-\Theta)$ with the unit ball $\mathbb{B} \subset Z$ is bounded. It is easy to see that the normality property of $\Theta$ implies its pointedness, but not visa versa.

It is well known (see, e.g., [11, Lemma 3.14]), that every minimal point of $\Xi$ is also a minimal point of the set $\Xi+\Theta$ while the reversed implication holds if $\Theta$ is pointed. The following lemma establishes similar (but somewhat different) relationships between super minimal points of the sets $\Xi$ and $\Xi+\Theta$.

Lemma 4.7 (relationships for super minimality). Let $\Xi \subset Z$ be a nonempty subset of a Banach space ordered by a convex cone $\Theta$. The following relationships hold:

(i) Every super minimal point of the set $\Xi+\Theta$ is a super minimal point of the set $\Xi$.

(ii) If the ordering cone $\Theta$ has the normality property, then every super minimal point of the set $\Xi$ is a super minimal point of the set $\Xi+\Theta$.

Proof. Assertion (i) follows directly from the definition of super minimality (3.1). To justify assertion (ii), fix an arbitrary super minimal point of the set $\Xi$. By definition (3.1) for any $z \in$ cone $(\Xi+\Theta-\bar{z}) \cap(\mathbb{B}-\Theta)$ there are $\xi \in \Xi, \theta \in \Theta$, and $t \geq 0$ such that $z=t(\xi+\theta-\bar{z}) \in \mathbb{B}-\Theta$. Thus we have $t(\xi-\bar{z}) \in \mathbb{B}-\Theta$ by the convexity of $\Theta$. Then the super minimality property (3.1) implies that

$$
t(\xi-\bar{z}) \in \text { cone }(\Xi-\bar{z}) \cap(\mathbb{B}-\Theta) \subset M B,
$$


and therefore we arrive at the inclusion

$$
z=t(\xi-\bar{z})+t \theta \in(M \mathbb{B}+\Theta) \cap(\mathbb{B}-\Theta)
$$

By the normality property of $\Theta$, the set $(\mathbb{B}+\Theta) \cap(\mathbb{B}-\Theta)$ is bounded, and hence there is $K>0$ such that $z \in K \mathbb{B}$, which gives

$$
\text { cone }(\Xi+\Theta-\bar{z}) \cap(\mathbb{B}-\Theta) \subset K \mathbb{B} \text {. }
$$

The latter justifies the super minimality of $\bar{z}$ for the set $\Xi+\Theta$ and thus completes the proof of the lemma.

Observe that the normality property of the ordering cone $\Theta$ in Lemma 4.7(ii) cannot be dropped. Indeed, consider the ordering cone $\Theta=\left\{z \in \mathbb{R}^{2} \mid z_{1} \geq 0\right\}$ and the set $\Xi=\{0\}$ in $\mathbb{R}^{2}$. It is easy to check that 0 is a super minimal point of $\Xi$ but not of the set $\Xi+\Theta$.

Now we are ready to derive subdifferential conditions for super minimizers to the original multiobjective problem (1.1) of our study.

Theorem 4.8 (subdifferential conditions for super minimizers to multiobjective problems). Let $(\bar{x}, \bar{z}) \in \operatorname{gph} F$ be a local super minimizer to problem (1.1), where both spaces $X$ and $Z$ are Asplund, and where $Z$ is partially ordered via (1.2) by a closed and convex cone $\Theta \subset Z$ satisfying the normality property. Assume that the epigraph of $F$ and the set $\Omega$ are locally closed around $(\bar{x}, \bar{z})$ and $\bar{x}$, respectively, that the cone $\Theta$ is SNC at the origin, and that the following requirements are fulfilled:

(a) either $F$ is partially SNEC at $(\bar{x}, \bar{z})$, or $\Omega$ is SNC at $\bar{x}$;

(b) the pair $\{F, \Omega\}$ satisfies the qualification condition

$$
\partial^{\infty} F(\bar{x}, \bar{z}) \cap(-N(\bar{x} ; \Omega))=\{0\},
$$

where both requirements (a) and (b) hold automatically when $F$ is ELL around $(\bar{x}, \bar{z})$.

Then taking $M>0$ from Definition 3.1 of local super minimizers and taking any $v^{*}$ from the closed unit ball of $Z^{*}$, we find $-z^{*} \in N(0 ; \Theta)$ with $\left\|z^{*}\right\| \leq M$ such that

$$
0 \in \partial F(\bar{x}, \bar{z})\left(z^{*}-v^{*}\right)+N(\bar{x} ; \Omega) .
$$

Assuming in addition that $\operatorname{int} N(0 ; \Theta) \neq \emptyset$, we get $-z^{*} \in \operatorname{int} N(0 ; \Theta)$ with $\left\|z^{*}\right\|=1$ and

$$
0 \in \partial F(\bar{x}, \bar{z})\left(z^{*}\right)+N(\bar{x} ; \Omega) .
$$

Proof. Considering the epigraphical multifunction $\mathcal{E}_{F}$ from (2.5) and using the normality property of the ordering cone $\Theta$, we conclude from Lemma 4.7(ii) that every local super minimizer $(\bar{x}, \bar{z}) \in \operatorname{gph} F$ to the original problem (1.1) is also a local super minimizer to the auxiliary multiobjective problem:

$$
\text { minimize } \mathcal{E}_{F}(x) \text { subject to } x \in \Omega,
$$

where the cost mapping $F$ is replaced by its epigraphical multifunction. Applying now Theorem 3.2 to the new problem (4.19) and taking into account the subdifferential constructions for set-valued mappings presented in Section 2 as well as the definitions and results for the partial SNEC and ECC properties given therein, we derive the qualification condition (4.16) and the optimality condition (4.17) of this theorem from the corresponding results of Theorem 3.2. The refined optimality condition (4.18) under the additional assumption on the nonempty interior of $N(0 ; \Theta) \neq \emptyset$ follows respectively from Theorem 3.7. 
Remark 4.9 (relationships between subdifferential and coderivative conditions for super minimizers). We can see that both the assumptions and the optimality conditions of the coderivative results in Section 3 and the subdifferential results in Section 4 are generally independent. The relationships between them more or less revolve around the relationships between the corresponding properties and graphs and epigraphs of set-valued mappings with values in partially ordered spaces and between the generalized normals to these sets. At the same time, the subdifferential results of Section 4 tend to be improvements of the corresponding coderivative results of Section 3 under additional assumptions. By Proposition 4.3 this is definitely the case for order semicontinuous cost mappings provided also that the ordering cone $\Theta$ has the normality property imposed in Theorem 4.8.

\section{References}

[1] Bao, T.Q., Gupta, P. and Mordukhovich, B.S. (2007), Necessary conditions in multiobjective optimization with equilibrium constraints, J. Optim. Theory Appl. 135, 179-203.

[2] Bao, T.Q. and Mordukhovich, B.S. (2007), Variational principles for set-valued mappings with applications to multiobjective optimization, Control Cybern. 36, 531-562.

[3] Bao, T.Q. and Mordukhovich, B.S. (2007), Existence of minimizers and necessary conditions in set-valued optimization with equilibrium constraints, Appl. Math. 6.

[4] Bednarczuk, E. (2007), Stability analysis for parametric vector optimization problems, Diss. Math. 442, 126 pp.

[5] Benson, H.P. (1979), An improved definition of proper efficiency for vector maximization with respect to cones, J. Math. Anal. Appl. 71, 232-241.

[6] Borwein, J.M. (1977), Proper efficient points for maximizations with respect to cones, SIAM J. Control Optim. 15, 57-63.

[7] Borwein, J.M. and Zhu, Q.J. (2005), Techniques of Variational Analysis, Canadian Mathematical Society Series 20, Springer, New York.

[8] Borwein, J.M. and Zhuang, D.M. (1993), Super efficientcy in vector optimization, Trans. Amer. Math. Soc. 338, 105-122.

[9] Geoffrion, A.M. (1968), Proper efficientcy and the theory of vector maximization, J. Math. Anal. Appl. 22, 618-630.

[10] Göpfert, A., Riahi, H., Tammer, C. and Zalinescu, C. (2003) Variational Methods in Partially Ordered Spaces, CMS Books in Mathematics 17, Springer, New York.

[11] Jahn J. (2004), Vector Optimization: Theory, Applications and Extensions, Series in Operations Research and Decision Theory, Springer, New York, 2004.

[12] Huang, H. (2007), The Lagrange multiplier rule for super efficiency in vector optimization, to appear in J. Math. Anal. Appl.

[13] Kuhn, H.W. and Tucker, A.W. (1951), Nonlinear programming, in Proceedings of the Second Berkeley Symposium on Mathematical Statictis and Probability, J. Neyman, ed., University of California Press, Berkeley, CA, pp. 481-492.

[14] Luc, D.T. (1989), Theory of Vector Optimization, Lecture Notes Econ. Math. Syst. 319, Springer, Berlin.

[15] Mordukhovich, B.S. (2006), Variational Analysis and Generalized Differentiation, I: Basic Theory, Grundlehren Series (Fundamental Principles of Mathematical Sciences) 330, Springer, Berlin. 
[16] Mordukhovich, B.S. (2006), Variational Analysis and Generalized Differentiation, II: Applications, Grundlehren Series (Fundamental Principles of Mathematical Sciences) 331, Springer, Berlin.

[17] Mordukhovich, B.S., Nam, N.M. and Yen, N.D. (2006), Fréchet subdifferential calculus and optimality conditions in nondifferentiable programming, Optimization 55, 685-708.

[18] Rockafellar, R.T. (1985), Maximal monotone relations and the second derivatives of nonsmooth functions, Ann. Inst. H. Poincaré: Analyse Non Linéaire 2, 167-184.

[19] Rockafellar, R.T. and Wets, R. J-B. (1998), Variational Analysis, Grundlehren Series (Fundamental Principles of Mathematical Sciences) 317, Springer, Berlin.

[20] Zheng, X.Y., Yang, X.M. and Teo, K.L. (2007); Super efficiency of vector optimization in Banach spaces, J. Math. Anal. Appl. 327, 453-460. 